\title{
Effects of Above and Below the Median of Fat Intake on Morbidity of Hyperglycemia of Middle Aged and elderly People in Zhang Fang Village, Fang Shan District, Beijing
}

\author{
Jia Wang* Zhongxin Hong \\ Department of Clinical Nutrition, Beijing Friendship Hospital, Capital Medical University, Beijing, China \\ *Corresponding author: wangjia830106@163.com
}

Received June 25, 2014; Revised July 15, 2014; Accepted July 22, 2014

\begin{abstract}
Objective To investigate the relationship between the fat intake and the morbidity of hyperglycemia(the fasting blood glucose over $6.1 \mathrm{mmol} / \mathrm{L}$, or the blood glucose one hour after the meal over $11.1 \mathrm{mmol} / \mathrm{L}$, or the blood glucose two hours after the meal over $7.8 \mathrm{mmol} / \mathrm{L}$, or the participants diagnosed diabetes previously) of middle aged and elderly people in Zhang Fang village, Fang Shan District, Beijing. Methods In $16^{\text {th }}$ July 2011, our nutrition department went to the Zhang Fang village Fang Shan District to do the free clinic. Totally 134 middle aged and elderly villagers were selected randomly. Our study was a cross-sectional study. We investigated the villagers about the diet intake, the life habit and disease history, measured the parameters of anthropometry and immediately glucose using Roche glucometer, and recorded the measure time. According to the glucose level, we categorized the villagers into hyperglycemia group and euglycemia group. According to the median of fat intake, we separated the villagers into relatively high fat group and relatively low fat group. We analyzed the differences between these groups, then used Logistic regression to analyze the indexes which can influence the blood glucose. Result The morbidity of hyperglycemia in our survey was $47.01 \%$. The morbidity of hyperglycemia in relatively high fat group was significantly higher than that in relatively low group. The intake of protein, dietary fiber, total energy and mixed dietary glycemic load in relatively high fat group were significantly higher than those in relatively low group group. The fat intake, energy intake, BMI and body fat in hyperglycemia group were significantly higher than those in euglycemia group. Logistic regression showed that high fat intake and increased BMI can raise the morbidity of hyperglycemia. Conclusion The morbidity of hyperglycemia of middle aged and elderly people in Zhang Fang Village was in high level. This phenomenon is associated with the high fat intake and increased BMI.
\end{abstract}

Keywords: hyperglycemia, fat intake, middle aged and elderly people, body mass index (BMI), life style

Cite This Article: Jia Wang, and Zhongxin Hong, "Effects of Above and Below the Median of Fat Intake on Morbidity of Hyperglycemia of Middle Aged and elderly People in Zhang Fang Village, Fang Shan District, Beijing.” Journal Name, vol. 2, no. 7 (2014): 406-410. doi: 10.12691/jfnr-2-7-13.

\section{Introduction}

The diabetes and prediabetes are highly prevalent in adult in China. The previous research showed that the agestandardized prevalence of diabetes and prediabetes were $9.7 \%$ and $15.5 \%$, respectively. The prevalence of diabetes among urban residents was higher than that among rural residents $(11.4 \%$ vs. $8.2 \%)$. And the prevalence of prediabetes among rural resident was higher than that among urban residents (16.1\% vs. $15.5 \%)$. This implied that the prevalence of diabetes in rural resident would higher than that in the urban resident some years later [1]. The reason caused diabetes or prediabetes includes obesity, the less activity, genetic factors and unbalanced dietary pattern. Recently, dietary macronutrient composition had received considerable attention [2,3]. The present study was conducted to explore the relationship between the daily intake of fat and the morbidity of hyperglycemia of middle aged and elderly people in Zhang Fang Village, a remote village in Beijing, and provide a basis for our diabetes prevention.

\section{Materials and Methods}

\subsection{Participants}

The department of clinical nutrition in Beijing Friendship Hospital provided free clinics for the rural residents in Zhang Fang Village, Fang Shan District in $16^{\text {th }}$ July 2011. The village committee arranged before. Every family in the village was required to attend 1 or 2 adults, 45 years of age or older. Our study was a crosssectional study. 150 villagers took part in our free clinics. We done the quickly glucose test and recorded the 
measure time. We measured anthropology indexes, surveyed the history of disease, and inquired the life style, such as smoking, drinking, sleeping, and exercise. Then we surveyed the daily dietary in detail. The inclusion criteria were as follows: age of $>45 \mathrm{y}$ and $<90 \mathrm{y}$, had no serious liver or kidney illness, had no cognitive impairment, had no mental abnormality, and gave written informed consent. The exclusion criteria were as follows: current pregnancy, intemperate, lineal relatives (such as father and son, mother and son, father and daughter or mother and daughter). From 150 participants screened for inclusion criteria, 134 participants met the criteria and enrolled in the study. 12 participants were age $<45 \mathrm{y}$ or $>90$ y. 4 participants had lineal relatives. The baseline of the participants in detail is showed in Table 1.

Table 1. Baseline characteristics of 134 participants of the study ( $n=134)$

\begin{tabular}{|c|c|c|}
\hline \multicolumn{2}{|l|}{ Item } & Data \\
\hline \multicolumn{2}{|c|}{ Number of participant (n) } & 134 \\
\hline \multicolumn{2}{|c|}{ Male person n(\%) } & $45(33.58)$ \\
\hline \multicolumn{2}{|c|}{ Female person n(\%) } & 89(66.42) \\
\hline \multirow{2}{*}{ Age } & minimum age maximum (years old) & $45 \sim 84$ \\
\hline & mean \pm SD (years old) & $61.39 \pm 10.81$ \\
\hline \multicolumn{2}{|c|}{ Drinking n(\%) } & 26(19.4) \\
\hline \multicolumn{2}{|c|}{ Smoking n(\%) } & $31(23.1)$ \\
\hline \multirow{2}{*}{ Exercise } & Light physical activity n(\%) & $91(68.0)$ \\
\hline & Medium and powerful physical activity n(\%) & $43(32.0)$ \\
\hline \multicolumn{2}{|c|}{ Exercise time (mean \pm SD, hours/day) } & $2.25 \pm 1.97$ \\
\hline \multicolumn{2}{|c|}{ Sleeping time (mean \pm SD, hours/day) } & $7.96 \pm 1.76$ \\
\hline \multirow{4}{*}{ BMI } & $<18.5 \mathrm{~kg} / \mathrm{m}^{2} \mathrm{n}(\%)$ & $6(4.5)$ \\
\hline & $18.5 \sim 23.9 \mathrm{~kg} / \mathrm{m}^{2} \mathrm{n}(\%)$ & $36(26.9)$ \\
\hline & $24 \sim 27.9 \mathrm{~kg} / \mathrm{m}^{2} \mathrm{n}(\%)$ & $55(41)$ \\
\hline & $>28 \mathrm{~kg} / \mathrm{m}^{2} \mathrm{n}(\%)$ & $37(27.6)$ \\
\hline \multicolumn{2}{|c|}{ Body Fat (mean \pm SD, \%) } & $25.9 \pm 11.1$ \\
\hline \multicolumn{2}{|c|}{$\mathrm{WHR}^{*}($ mean $\pm \mathrm{SD})$} & $0.910 \pm 0.056$ \\
\hline \multicolumn{2}{|c|}{$\mathrm{TSF}^{\#}($ mean $\pm \mathrm{SD}, \mathrm{mm})$} & $19.3 \pm 8.3$ \\
\hline \multicolumn{2}{|c|}{ Energy intake (mean \pm SD, kcal) } & $1963.6 \pm 631.8$ \\
\hline \multicolumn{2}{|c|}{ Carbohydrate intake (mean \pm SD, g) } & $339.4 \pm 119.3$ \\
\hline \multicolumn{2}{|c|}{ Protein intake (mean \pm SD, g) } & $54.4 \pm 22.6$ \\
\hline \multicolumn{2}{|c|}{ Fat intake (mean $\pm \mathrm{SD}$, g) } & $39.6 \pm 21.7$ \\
\hline \multicolumn{2}{|c|}{ Dietary fiber intake (mean $\pm \mathrm{SD}, \mathrm{g}$ ) } & $11 \pm 6$ \\
\hline
\end{tabular}

* WHR:waist hip rate

\#TSF: triceps skin-fold

The study was approved by the ethical committee of Beijing Friendship Hospital of Capital Medical University. And the participants had all written the informed consent.

\subsection{Measurements}

The blood glucose was measured by using a portable digital glucometer and test paper (ACCU-CHEK® Active, Model:GC, is a trademark of Roche, made in China), taking the middle finger tip blood of the right hand. Data about the glucose and the glucose measure time, such as fasting blood glucose, or the blood glucose one hour after the meal, or the blood glucose two hours after the meal, were recorded. Then investigators asked the participants whether or not to be diagnosed diabetes previously and recorded.

Diagnosed standard [4]: the fasting blood glucose over $6.1 \mathrm{mmol} / \mathrm{L}$, or the blood glucose one hour after the meal over $11.1 \mathrm{mmol} / \mathrm{L}$, or the blood glucose two hours after the meal over $7.8 \mathrm{mmol} / \mathrm{L}$, or the participants diagnosed diabetes previously were classified into the hyperglycemia group. The other participants were classified into the euglycemia group.

Body weight was measured while the participants were wearing light clothing and no shoes. Height without shoes was measured by using a stadiometer. And body mass index (BMI) was calculated as weight divided by the square of height. We used a tape to measure the waist girth as the narrowest circumference between the bottom of the rib cage and the iliac crest, the hip girth as the widest circumference, and the biceps circumference as the fullest upper arm circumference. The waistline hipline rate (WHR) was calculated as waistline divided by the hipline. We used skinfold instrument to measure triceps skinfold thickness. The arm muscle circumference was calculated as upper arm circumference minus 3.14 times the triceps skinfold.

We used portable bioelectrical impedance analyzer (Jas technology trade co., LTD, Japan) to measure the body fat. The subjects, with arms straight and body axis was 90 degrees, hold the two sides of the electrode with both hands forefinger and thumb force, then read and recorded the data after 15 seconds.

The methods of lifestyle survey: (1)drinking: record drinking or not, and the capacity for liquor. (2)smoking: record smoking or not, and the smoking number per day. 
(3)exercise: record the daily activity time and the activity intensity. (4)sleeping: record the daily sleeping time.

Dietary survey method: 24-hour dietary retrospective method. 24-hour generally refer to 24 hours from the last meal to eat began to push forward for 24 hours. Select continuous 3 days, record the food variety and intake.

We calculated the mixed dietary glycemic index (GI) and the mixed dietary glycemic load (GL). The GI value of each food item was defined as the percentage of postprandial blood glucose response of the food compared with white bread as the standard food. The overall dietary GI was calculated by adding the products of the carbohydrate content of each food, multiplying this sum by its GI, multiplying this number by the average number of servings consumed per day, and then dividing this by the total intake of available carbohydrate. The total dietary GL of each food was calculated by multiplying the quantity of each food item consumed per day by the amount of carbohydrate contained in a specified serving size of the food and its corresponding GI value (considering glucose as the reference food). Total GL was then estimated by summing the values for all carbohydrate-containing foods, whereas total GI was calculated by dividing the total dietary GL by the total available carbohydrate intake.

\subsection{Statistical Analysis}

Data are presented as means \pm SDs. Potential withingroup treatment effects on nutrient intake, index diet GI, index diet GL, age, anthropology index were analyzed by using Student's t test for data. A chi-square test was used to compare discrete variables. Multiple backward stepwise regression analysis was used to study the independent variables able to predict hyperglycemia and nutrient intake during the study. The results of the latter are shown as $\beta$ coefficients. The 2-tailed significance threshold was set as $\mathrm{P}<0.05$. All statistics were calculated with the use of the STATISTICAL PACKAGE FOR SOCIAL SCIECES (SPSS for Windows, version 17.0; SPSS, Chicago, IL).

\section{Results}

From 150 participants screened for inclusion criteria, 134 participants met the criteria and enrolled in the study. Table 1 describes the general condition, living habit, anthropometric and food intake of the research objects.

In the 134 research objects, 24 objects who were diagnosed diabetes before. In the objects who were not diagnosed diabetes, 27 objects were fasting glucose abnormal, 1 object was 1-hour after meal blood glucose abnormal, and 11 objects were 2-hour after meal glucose abnormal. The percentage of prior diagnosis of diabetes was $17.91 \%$. The percentage of the new discovery hyperglycemia was $29.10 \%$. The percentage of total hyperglycemia was $47.01 \%$.

The research objects were separated into two groups, according to the median level of the fat intake everyday, $33.8 \mathrm{~g} / \mathrm{d}$. The objects who daily fat intake more than $33.8 \mathrm{~g} / \mathrm{d}$ were st into relatively high fat intake group (RHF group), and who daily fat intake less than $33.8 \mathrm{~g} / \mathrm{d}$ were set into relatively low fat intake group (RLF group). In RLF group, there was 24 cases of hyperglycemia, and the percentage of hyperglycemia was $35.82 \%$. In RHF group, there were 39 cases of hyperglycemia, and the percentage of hyperglycemia was $58.21 \%$. Compared the incidence of hyperglycemia between RLF group and RHF group, the incidence of hyperglycemia in RHF group was significantly higher than that in RLF group $\left(\chi^{2}=6.740\right.$, $\mathrm{P}=0.015$ ).

Compare the intake of carbohydrate, protein, dietary fiber and energy between the RHF group and RLF group. Compared the daily intake of protein between RLF group and RHF group, the intake of protein in RLF group (43.28 $\pm 13.45 \mathrm{~g})$ was lower than that in RHF group (65.42 \pm $24.58 \mathrm{~g})(\mathrm{t}=6.469, \mathrm{P}<0.01)$. Compared the daily intake of dietary fiber between RLF group and RHF group, the intake of dietary fiber in RLF group (9.5 \pm 4.35 g) was lower than that in RHF group $(12.45 \pm 6.66 \mathrm{~g})(\mathrm{t}=3.030, \mathrm{P}<0.01)$. Compared the daily intake of total energy between RLF group and RHF group, the intake of total energy in RLF group (1720.72 $\pm 535.75 \mathrm{kcal})$ was lower than that in RHF group $(2206.48 \pm 630.58 \mathrm{kcal})(\mathrm{t}=4.805, \mathrm{P}<0.01)$. While the intake of carbohydrate has no significantly different between two groups $(t=1.78, P=0.078)$. Compared the mixed dietary glycemic index (GI) and the mixed dietary glycemic load (GL) between RHF group and RLF group, the mixed GL in RHF group (87.25 \pm 27.82) was significantly higher than GL in RLF group (80.86 \pm 32.83$)$ $(t=2.216, \quad P=0.026)$. While the mixed GI has no significantly different between two groups $(\mathrm{t}=1.115$, $\mathrm{P}=0.236$ ). The intake of protein, dietary fiber, energy and mixed GL in RHF group was higher than those in RLF group. It showed that with the increase of fat intake, the total energy intake increased caused an increased incidence of hyperglycemia.

Compare the age, gender, life style, anthropometry between RHF and RLF groups, two groups were comparable. The age, sex, life style had no significantly difference between two groups $(\mathrm{P}>0.05)$. And the anthropometry had no difference also $(\mathrm{P}>0.05)$. It showed that the incidence of hyperglycemia between two groups was not associated with changing the composition of human body.

In our study, the dietary habit of the villagers is monotonous and mainly staple diet. We separated the objects into two groups, hyperglycemia group and euglycemia group. Using student's t analysis to compare the intake of carbohydrate, fat, protein dietary fiber, total energy and mixed GI and GL, it showed that between hyperglycemia group and euglycemia group the intake of fat had significant difference $(t=2.930, \mathrm{P}=0.036)$, the intake of total energy had significant difference $(t=2.251$, $\mathrm{P}=0.023$ ), and others indexes had no significant difference $(\mathrm{P}>0.05)$.

We used $\chi^{2}$ test to compare two groups in gender and living habit. There were no significantly difference between two groups about gender, drinking, smoking, exercise and sleeping $(P>0.1)$. Then we used student's $t$ test to compare the two groups in age and anthropometry. The age, waist hip rate and triceps skinfold thickness had no significant difference between hyperglycemia group and euglycemia group $(P>0.1)$. The body mass index had significant difference between two groups $(t=-1.993$, $P=0.043$ ) , and body fat also had difference between two groups $(t=-1.858, P=0.047)$. 
By using univariate analysis, the intake of fat and energy, the BMI and BF index had significant difference between euglycemia group and hyperglycemia group. Then we use logistic regression analysis to analyze which index can influence the blood glucose significantly. In the end, the intake of fat and BMI were drew into the regression equation. The intake of fat and BMI were significant correlated with the blood glucose. And with the increasing of fat intake and BMI, the incidence of hyperglycemia was increased. Table 2 describes the data in detail.

Table 2. The parameter of regression analysis

\begin{tabular}{cccc}
\multicolumn{4}{c}{ Table 2. The parameter of regression analysis } \\
\hline & $\mathrm{B}$ & Wald & $\mathrm{P}$ \\
\hline Intake of fat & 0.024 & 3.986 & $\mathbf{0 . 0 4 6}$ \\
Intake of energy & 0.000 & 0.041 & 0.839 \\
BMI & 0.159 & 3.891 & $\mathbf{0 . 0 4 7}$ \\
BF & -0.026 & 1.172 & 0.279 \\
Constant & -3.232 & 5.322 & 0.021 \\
\hline
\end{tabular}

\section{Discussion}

The diabetes and prediabetes are highly prevalent in adult in China. The previous research showed that the agestandardized prevalences of diabetes and prediabetes were $9.7 \%$ and $15.5 \%$, respectively. The prevalence of diabetes was higher among urban residents than among rural residents. And the prevalence of prediabetes was higher among rural resident than among urban residents. This implied that the prevalence of diabetes in rural resident would higher than that in the urban resident soon [1].

The present study surveyed the elderly villagers in Zhan Fang Village Fang Shan District randomly about the life style, anthropometry, blood glucose and dietary habit. The study showed that the incidence of hyperglycemia was 47.01\% which was higher than paper reported before. In the 134 villagers, 63 villagers were diagnosed hyperglycemia, and 39 villagers were new-found, which percentage was $29.1 \%$. This data is likely to underestimate the incidence of hyperglycemia in this village. The reasons why the data was underestimated are following: (1)the present study measure the fasting glucose, or the blood glucose after eating one-hour, or the blood glucose after eating two-hours. We did't measure the three time blood glucose for each participant. So when the fasting blood glucose is in the normal range, we put it into the euglycemia group, but the blood glucose after eating onehour or two-hours maybe abnormal. This participant is diagnosed as impaired glucose tolerance. We should put it into the hyperglycemia group; put it into the euglycemia group. (2)another case, when the blood glucose after eating is in the normal range, we put it into the euglycemia group, but the fasting blood glucose maybe abnormal. This participant is diagnosed as impaired fasting glucose. We should put it into the hyperglycemia group, put it into the euglycemia group. In these two cases, we both underestimate the incidence of hyperglycemia.

After analysis of the survey data, the incidence of hyperglycemia in RHF group is significantly higher than that in RLF group. Between these two groups, the intake of carbohydrate has no significant difference, while the intake of protein, total energy, dietary fiber and mixed GL have significant difference. Compare the age, gender, life style, anthropometry between RHF and RLF groups, two groups were comparable. The age, sex, life style had no significantly difference between two groups. And the anthropometry had no difference also. It showed that the incidence of hyperglycemia between two groups was not associated with changing the composition of human body.

In our study, the dietary habit of the villagers is monotonous and mainly staple diet. Analysis above showed that different fat intake might influent the hyperglycemia. But the influence of various nutrients intake on the incidence of hyperglycemia is not clear. So then we separated the objects into another two groups, hyperglycemia group and euglycemia group. Using student's t analysis to compare the intake of carbohydrate, fat, protein dietary fiber, total energy and mixed GI and GL, it showed that between hyperglycemia group and euglycemia group the intake of fat and total energy had significant difference, and others had no significant difference. We used student's t test to compare the two groups in age and anthropometry, and used $\chi^{2}$ test to compare them in gender and living habit. The age, gender, living habit, waist hip rate and triceps skinfold thickness had no significant difference between hyperglycemia group and euglycemia group. The body mass index and body fat had significant difference between two groups.By using univariate analysis, the intake of fat and energy, the BMI and BF index had significant difference between euglycemia group and hyperglycemia group. Then we use logistic regression analysis to analyze which index can influence the blood glucose significantly. In the end, the intake of fat and BMI were drew into the regression equation. The intake of fat and BMI were significant correlated with the blood glucose. And with the increasing of fat intake and BMI, the incidence of hyperglycemia was increased.

Zhang Fang Village, Fang Shan District is a remote mountainous area of Beijing. The economy is relatively backward. The dietary survey of the villagers showed that, the diet is relatively monotonous. The basic dietary pattern is staple food and salted vegetables. The villagers who living condition are better can eat some meat and fat foods. But the carbohydrate intake is not decreased due to the increase of fat intake. This dietary pattern due to the increasing of total energy in relatively high fat group. From our research, although there is no significant difference of mixed GI between two groups, there is significant difference of mixed GL between two groups. Because the carbohydrate intake in RHF group are not decreased, and the total energy are also not decreased. These reasons cause the increased incidence of hyperglycemia in the RHF group. Even if the protein and dietary fiber intake in the RHF group were also significantly increase than that intake in the RLF group, but the benefit of these two nutrients in diabetes prevention and treatment of hidden are apparently concealed. The research showed that, there was no significant difference of the anthropometry and living habits between two groups. It means that the increased fat intake caused the higher incidence of hyperglycemia are by changing the BMI, BF, WHR, TSF and some living habits.

Further, we applied logistic regression to analyze the factors which cause the hyperglycemia. In the end, only two factors, fat intake and BMI were introduced into the 
regression equation. It means that, in this village, the occurence of hyperglycemia was related to the more fat intake and the higher BMI.

In the present study, the fat intake of the villagers is not high, mean of $39.6 \mathrm{~g}$ per day. In the RHF group, the mean fat intake is 54.42g per day. This fat intake is not higher than the resident reference intake. But the villagers in the RHF group didn't take low carbohydrate with taking more fat food, and take more energy and increase the mixed GL. This caused the hyperglycemia. The pathogenesis maybe due to increasing fat intake, but carbohydrate intake did not reduce, resulting in increasing energy intake, caused lipotoxicity. Lipotoxicity refer to the concentration of free fatty acid increased or higher fat content in the cell to the role of diabetes formation, the main working part of the liver, muscles and pancreas. A large number of studies have shown that under the environment of high fat islet beta cells dysfunction is closely related to the onset of type 2 diabetes, the beta cells apoptosis is increased [8]. Some scholars studied the lipotoxicity. Oral fat load test was given between 8:00-9:00h after a 12-h fast and a 3day abstention from alcohol intake. The test drink consisted of $350 \mathrm{ml}$ whipping cream (35\% fat), which contained $1147 \mathrm{kcal}$, of which $12 \%$ was from protein, $20 \%$ from carbohydrate, and $68 \%$ from fat. It had $472 \mathrm{mg}$ cholesterol and a polyunsaturated/saturated ratio of 0.06 . The fat load mixture was consumed within $10 \mathrm{~min}$. After the ingestion of the fat load, subjects were only allowed to drink water during the following $12 \mathrm{~h}$. Blood samples were drawn before and 3, 6, 9 and $12 \mathrm{~h}$ after the fat load. Observe 12 hours blood glucose, the blood lipid and inflammatory cytokines, cell adhesion molecules and other changes. A total of 286 cases of healthy individuals (with 141 cases male) joined in this test. The results shown that, fat load after $3 \mathrm{~h}$, 6h, 9h blood glucose were $3.26 \%, 4.35 \%$ and $1.09 \%$, respectively, only $12 \mathrm{~h}$ blood glucose fell slightly. Meanwhile, IL-6, TNF-a, hsCRP and cell adhesion molecules increased significantly, and prior to elevated plasma triglycerides. It shown that fat excessive intake can rapid rise the blood glucose [9]. Pan $\mathrm{MH}$, etc. [10] study found that high fat diet can damage the insulin signaling pathway, so as to elevate the blood glucose level. The American Diabetes Association (ADA) the increasing of the fat intake and energy consumption is the risk factor of increasing incidence of diabetes [11].

The BMI and BF in hyperglycemia group are higher than those in euglycemia group. It because that in hyperglycemia group, the fat intake increased without decreased the carbohydrate intake, it caused the total energy intake is higher than the body consumption. This cause the body weight gain and body fat increased. Meanwhile, the increased body weight and body fat, in turn, will reduce the insulin sensitivity. It will cause the insulin resistance, and cause the pathoglycemia. Study had found that, although the fat intake can't directly increase the risk of type 2 diabetes, it can influence the blood glucose through increasing the body weight and body fat [11].

Our study showed that, the people who fat intake relatively more should be taken to properly reduce the intake of carbohydrate in increasing the fat intake to ensure the total energy intake are not overweight, and meanwhile reduce the mixed GL, to control the blood glucose and avoid the occurrence of hyperglycemia. To avoid hyperglycemia in the general population, decreasing the fat intake, controlling the body weight, making the BMI in the normal range, will avoid the occurrence of hyperglycemia.

The present study found that total energy and fat intake affect the incidence of hyperglycemia together, and there are no literatures which study the fat intake or energy intake alone with hyperglycemia or the occurrence of diabetes. We will design the next research to discuss the influence of hyperglycemia or diabetes with changing the fat intake alone.

\section{Acknowledgments}

I would like to thank the following institutions and people who support our research: Zhang Fang Village committee, Zhang Fang villagers, the whole party member and two graduated students in the department of clinical nutrition in Beijing Friendship Hospital. And also thanks to the party of Beijing Friendship Hospital.

\section{References}

[1] Yang W, Lu J, Weng J, et al. Prevalence of diabetes among men and women in China. N Engl J Med, 2010; 362(12): 1090-101.

[2] Kevin D. Ballarda, Erin E. Quanna, Brian R. Kupchaka, et al. Dietary carbohydrate restriction improves insulin sensitivity, blood pressure, micro vascular function, and cellular adhesion markers in individuals taking statins. Nutrition Research 2013; 33, (11), 905-912

[3] Somayeh Rajaie M.Sc., Leila Azadbakht Ph.D., Majid Khazaei Ph.D., et al. Moderate replacement of carbohydrate by dietary fats affects features of metabolic syndrome: A randomized crossover clinical trial. Nutrition, 2014; 30: 61-68.

[4] Department of Noncommunicable Disease Surveillance. Definition, diagnosis and classification of diabetes mellitus and its complications: report of a WHO consultation. Part 1. Diagnosis and classification of diabetes mellitus. Geneva: World Health Organization, 1999.

[5] Ghazaleh Eslamian, Mahsa Jessri, Bahareh Hajizadeh, et al. Higher glycemic index and glycemic load diet is associated with increased risk of esophageal squamous cell carcinoma: a casecontrol study. Nutrition Research, 2013; 33(9):719-725.

[6] F.S. Atkinson, K. Foster-Powell, J.C. Brand-Miller. International tables of glycemic index and glycemic load values: 2008.Diabetes Care, 2008; 31 (12): 2281-2283.

[7] Karin Klack, Eloisa Bonfa, Eduardo Ferreia Borba Neto. Diet and nutritional aspects in systemic lupus erythematosus. Rev. Bras. Reumatol. 2012; 52(3): 384-408.

[8] Xiang Wei, Huang Zhongxin, Shi Bingyin. The research progress of the dysfunction of lipotoxicity and islet beta cell. Journal of Postgraduates of Medicine (Medicine Edition), 2004; 7(5): 44-45.

[9] G. Derosa, I. Ferrari, A. D’Angelo, et al. Oral fat load effects on inflammation and endothelial stress markers in healthy subjects. Heart Vessels 2009; 24: 204-210.

[10] Pan MH, Lai CS, Tsai ML, et al. Chemoprevention of nonalcoholic fatty liver disease by dietary natural compounds. Mol Nutr Food Res. 2013, 00: 1-25.

[11] Li hongjin, Zhao wen, Zuan Wenwen. 2010 the American Diabetes Association diabetes treatment guidelines. Chin J Stroke, 2011, 6(4): 316-318.

[12] VanDam RM, Willett WC, Rimm EB, et al. Dietary fat and meat intake in relation to risk of type 2 diabetes in men. Diabetes Care, 2002; 3: 417-424. 